\section{COMBINATION OF CLICK CHEMISTRY-BASED SQ3370 WITH IMMUNOTHERAPIES ENHANCES ANTITUMOR EFFECT IN MURINE TUMORS WITH MINIMIZED SYSTEMIC TOXICITY}

Amir Mahmoodi*, Sangeetha Srinivasan, Michael Zakharian, Nathan Yee, Jesse McFarland, Jose Mejia Oneto. Shasqi, Inc., San Francisco, CA, United States

Background Immunotherapies have enabled unprecedented durable responses in solid tumors. However, they benefit only a subset of patients and have varying response rates across tumor types. Conversely, conventional chemotherapies are effective in a larger patient population, but lack specificity and result in severe dose-limiting systemic toxicities. While anthracyclines such as doxorubicin (Dox) may provide added benefit by inducing anti-tumor immune activation, ${ }^{1}$ their overall effect is limited by cumulative dose cardiotoxicity. ${ }^{2}$ Here, we present the Click Activated Protodrugs Against Cancer (CAPAC) platform that activates cytotoxic protodrugs at the tumor using click chemistry. The CAPAC platform is agnostic to tumor characteristics such as biomarker expression and can be applied in a variety of tumor types. SQ3370 (lead candidate of CAPAC) consists of an intratumorally-injected biopolymer that can activate multiple doses of a systemic protodrug to release active Dox at the tumor. SQ3370 enables a 19-fold increase over the conventional Dox dose in mice with minimal systemic toxicity. ${ }^{3}$ In tumor-bearing mice, SQ3370 improved overall survival, T-cell infiltration, and induced a robust antitumor response against both biopolymer-injected and noninjected lesions, ${ }^{4}$ suggesting that SQ3370 promotes anti-tumor immune activation. This makes SQ3370 an ideal candidate for combination approaches with immunotherapies.

Methods SQ3370 treatment is described in figure 1. Immunocompetent mice were inoculated with MC38 tumors. SQL70 was given intratumorally; SQP33 was given intravenously as five daily doses. Immune adjuvants such as toll-like receptor (TLR) agonists or STING agonist were coadministered with SQ3370. Saline and conventional Dox served as controls.

Results Combining SQ3370 with endosomal TLR agonists such as poly I:C (TLR3a), imiquimod (TLR7a) or CpG ODN (TLR9a) resulted in improved tumor growth inhibition and survival in MC38 tumor-bearing mice compared with monotherapy. Combination with STING agonist, ADU-S100, also enhanced antitumor efficacy. Body weight data suggests minimal impact of the monotherapy or combination therapy on systemic toxicity.

Conclusions CAPAC represents a new therapeutic modality to treat solid tumors by expanding the pharmacological capabilities of cancer drugs with known efficacy and experimental drugs. SQ3370, CAPAC's lead candidate, improves safety and efficacy as compared to conventional Dox, and combination of SQ3370 with immunotherapy shows enhanced benefit. SQ3370 is being evaluated in a Phase I study in advanced solid tumors (NCT04106492).

Acknowledgements This work was supported by Shasqi and the National Institutes of Health.
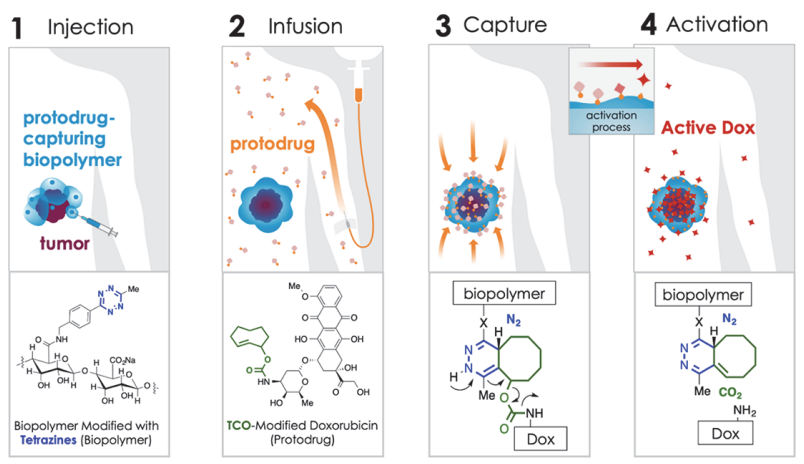

Abstract 695 Figure 1 Illustration and Molecular Description of SQ3370. SQ3370 consists of 2 components and utilizes Shasqi's proprietary click chemistry-based CAPAC platform. First, SQL70, a tetrazine-modified sodium hyaluronate biopolymer, is injected at the tumor site. Then, SQP33, a trans-cyclooctene (TCO)-modified protodrug of doxorubicin (Dox), is given systemically as 5 daily doses. SQP33 protodrug has attenuated toxicity and is converted to active Dox by SQL70 biopolymer through an efficient covalent reaction between tetrazine and TCO moieties

\section{REFERENCES}

1. Mattarollo SR, Loi S, Duret H, Ma Y, Zitvogel L, Smyth MJ. Pivotal role of innate and adaptive immunity in anthracycline chemotherapy of established tumors. Cancer Res 2011;71:4809-20.

2. Quintana RA, Banchs J, Gupta R, Lin HY, Raj SD, Conley A, Ravi V, Araujo D, Benjamin RS, Patel S, Vadhan-Raj S. Early evidence of cardiotoxicity and tumor response in patients with sarcomas after high cumulative dose doxorubicin given as a continuous infusion. Sarcoma 2017:7495914.

3. Wu K, Yee NA, Srinivasan S, Mahmoodi A, Zakharian M, Oneto JM, Royzen M Click activated protodrugs against cancer increase the therapeutic potential of chemotherapy through local capture and activation. Chem Sci 2021;12:1259-71.

4. Srinivasan S, Yee NA, Wu K, Zakharian M, Mahmoodi A, Royzen M, Mejía Oneto JM. SQ3370 activates cytotoxic drug via click chemistry at tumor and elicits sustained responses in injected and non-injected lesions. Adv Ther 2021;4:2000243.

Ethics Approval This study, project number: SSQ-FFS-ON20210225-01V3 1, was approved by the Institutional Animal Care and Use Committee (IACUC) of the vendor, following the guidance of Association for Assessment and Accreditation of Laboratory Animal Care (AAALAC), accreditation number 001516.

http://dx.doi.org/10.1136/jitc-2021-SITC2021.695 